\title{
A Novel Approach towards the Identification of plant genes, Bioinformatics Analysis and Role of EIN3 and EIL1 in plants
}

\author{
Syed Abu Bakr Haider Bukhari ${ }^{1}$, Muhammad Waqas Akhtar ${ }^{2}$, Shuja Ur Rehman ${ }^{3}$, Waseem Akhtar ${ }^{4}$, Hamza Rafeeq $^{3 *}$
} Alyan Ashraf ${ }^{5}$, Muhammad Zeeshan Talib ${ }^{1}$, Naveed Aslam ${ }^{1}$

${ }^{1}$ Department of Botany, University of Agriculture, Faisalabad, Pakistan

${ }^{2}$ Department of Plant Pathology, University of Agriculture, Faisalabad, Pakistan

${ }^{3}$ Department of Biochemistry, University of Agriculture, Faisalabad, Pakistan

${ }^{4}$ Institute of Soil and Environmental Sciences, University of Agriculture, Faisalabad, Pakistan

${ }^{5}$ Centre of Agricultural Biochemistry and Biotechnology (CABB), University of Agriculture, Faisalabad, Pakistan

DOI: $\underline{10.36348 / \mathrm{sijb} .2020 . \mathrm{v} 03 \mathrm{i} 10.001}$

| Received: 15.09 .2020 | Accepted: 24.09.2020 | Published: 16.10.2020

*Corresponding author: Hamza Rafeeq

\section{Abstract}

One of the most important parameter in elongation of cotton fibre is the biosynthesis of ethylene. EIN3 and EIL1 (Ethylene Insensitive3/Ethylene Insensitive 3) are the main transcriptional factors that are specifically playing an important role in signal transduction in cotton. Expression analysis of the EIN3/EIL1 genes involved in development of cotton fibre investigated out through RT-qPCR. Further, STRINGTIE is a tool that is use for measuring the different levels of expression. ClustalW method is a method that is used to determine the sequences of amino acids of EIN3/EIL1 genes by aligning by using the sequences. KEGG (Kyoto Encyclopedia of Genes and Genomes) pathways of the EIN3/EIL1 genes is also bioinformatics tools that is functionally use for classification and characterization of genes at functional level with using the principles of functional enrichment analysis. Biological database such as STRING is designed for to construct the specific PPI networks of EIN3/EIL1 due to known PPIs and analysis to check interactions among protein. Bioinformatics tool such as BLASTP use for identification of transcription factors of EIN3/EIL1genes. Biological database such as Plant CARE is especially used to analyze the cis-elements in specific sequences of promoters of EIN3/EIL1 that are especially involved in transcription of genes.

Keywords: EIN3 gene, EIL1 gene, Gene knockout, Cotton Fibre, Ethylene.

Copyright ( $) 2020$ The Author(s): This is an open-access article distributed under the terms of the Creative Commons Attribution 4.0 International License (CC BY-NC 4.0) which permits unrestricted use, distribution, and reproduction in any medium for non-commercial use provided the original author and source are credited.

\section{INTRODUCTION}

Ethylene has a significant role in the growth of plants such as maintaining the elongation processes as well as development of cotton fibre [1]. One of the most important parameter in elongation of cotton fibre is the biosynthesis of ethylene. Production of ethylene in appropriate amount significantly increases the fibre development in Gossypium hirsutum. Inositol synthase and expansion are involved in fibre development in cotton. Ethylene overall controls the gene expression of inositol synthase and expansion. Fatty acids that are long chain in nature also involved in elongation of cotton fibren [2].

EIN3 and EIL1 (Ethylene Insensitive3/Ethylene Insensitive 3) are the main transcriptional factors that are specifically playing an important role in signal transduction in cotton. Both these factors are involved in regulating the signaling responses induced by ethylene. EIN3 and EIL1 are mainly involved in regulating the transcription by activating the other factors that involved in transcription such as ethylene response factor [3].

EIN3 and EIL1 make strong interactions with other hormones as well as transcriptional factors by inducing the cellular signaling and thus regulating the gene expression in plants [4]. EIN3 and EIL1 also regulate the signal transduction in Arabidopsis by regulating the expression of GCC box. EIN3 binding Fbox proteins have a significant role in regulating the responses induced by ethylene in Arabidopsis [5]

The structure of EIN3/EIL1 comprises mainly two biochemical features in relation to sequence of amino acids that maintain the functional activities of these proteins Firstly, EIN3/EIL1 proteins contain special sequences of special amino acids at the aminoterminal that are highly conserved. Secondly, the sequences of the carboxy terminal of EIN3/EIL1 
proteins are lower and less conserved as compared to the sequences of the amino terminal $[6,7]$.

There are several functions of EIN3/EIL1genes for regulation of biochemical pathways in some plants such as Arabidopsis and tomato. Molecular mechanism of transcription is initiated by responses of ethylene and regulation of transcription is crucial in Arabidopsis[8]. Biochemical roles as well as cellular properties of genes associated with EIN3 and EIL1 have been studied in various plants such as rice, tobacco, tomato and Arabidopsis [9].

EIN3 plays an important role in regulation of transcription in Arabidopsis. EIN3 also acts as a main transcriptional regulator in response to sulphur metabolism in Arabidopsis. EIN3/EIL1genes show a relationship with ethylene as well as sulphur metabolism in plants. Nicotiana tabacum EIL2 is involved in regulation of expression of some genes in tomato by binding with the UP9C promoter [10]. The role of EIN3 and EIL1genes in the development of cotton fibre has not completely described. Molecular regulation of EIN3 and EIL1genes at transcriptional level in cotton remains unclear. The molecular mechanism and biochemical process of the development of cotton fibre in which EIN3 and EIL1genes play a main role remains unclear. Fibre mutants are the main useful source for describing the molecular mechanism in the development of cotton fibre.

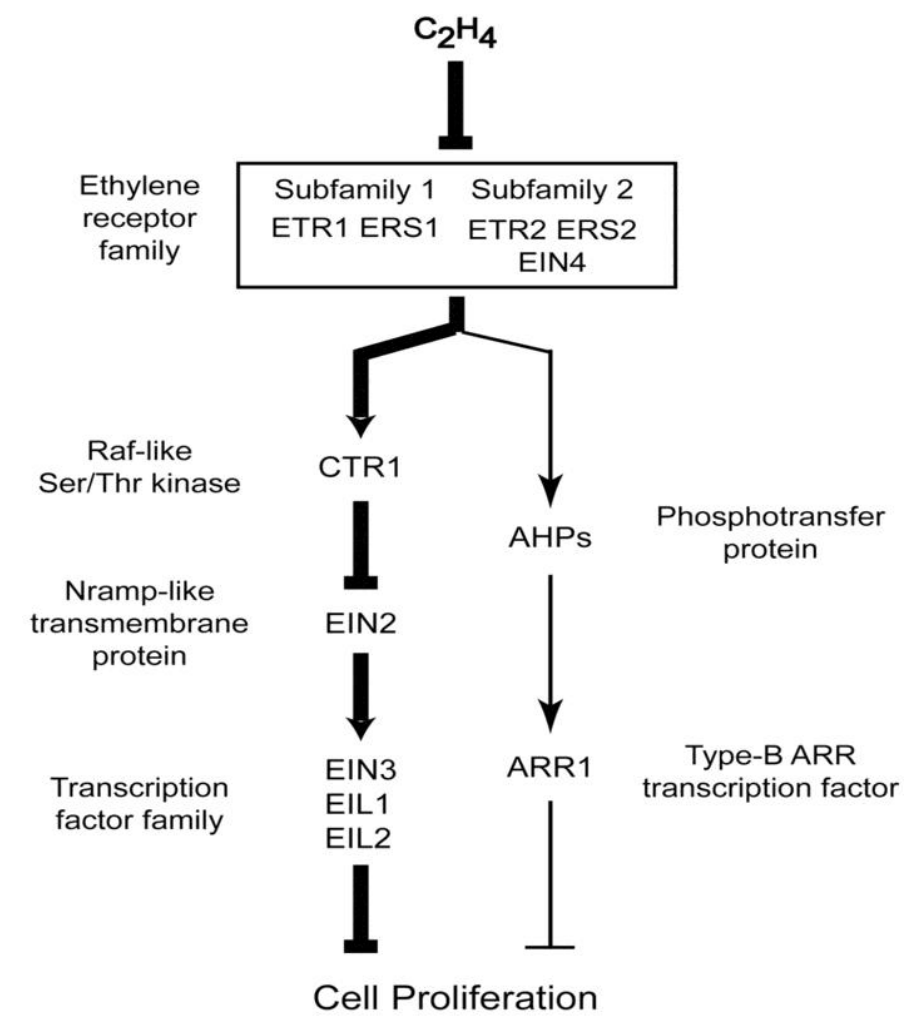

Fig-1: Molecular regulation of EIN3 and EIL1genes at transcriptional level

The aims of this review article investigated aspects of the phylogenetic analysis for determination of the sequences of amino acids of EIN3/EIL1 genes, investigate the gene expression analysis of EIN3/EIL1 genes involved in development of cotton fibre through RT-qPCR, functional information about EIN3/EIL1 genes through conducting the bioinformatics analysis also discussed the interaction among EIN3/EIL1 genes through constructing the specific PPI networks of EIN3/EIL1.

\section{Molecular Analysis of EIN3/EIL1 genes}

Regulation of EIN3/EIL1 levels via the control of the protein stability by EBF1/2 is a pivotal mechanism of EIN3/EIL1 adjustment in ethylene signaling. Simultaneously, it can be affected by environmental factors resulting in a modulation of transcriptional response to ethylene. Plants germinating in the darkness assume a light-regulated developmental program known as skotomorphogenesis, which phenotypically results in rapid hypocotyl elongation, small closed chlorotic cotyledons, and apical hook formation [8].

Plant materials such as upland cotton that functionally growing under natural conditions and cotton flowers according to design of the fibers of the cotton labeled properly [11]. Cotton flowers tied carefully before for self pollination. All cotton samples collected according to following the instructions following by Perkin Elmer [12]. The samples of cotton fibre frozen carefully under liquid nitrogen. All other 
sample collection and preparation of samples carried out under the natural conditions following the fields of the cotton growing of the cotton [13].

Applied Biosystems-7500 RT-PCR detection systems use for samples analysis carried out for the RTqPCR experimental investigation (Applied Biosystems,USA). Specific primers designed for EIN3/EIL1 genes that uses for the experiments conducting through the RT-qPCR experimentation. Three replication undergoing the taken for every sample during the experimental investigation. Expression analysis of the EIN3/EIL1 genes involved in development of cotton fibre investigated out through RT-qPCR. Further, STRINGTIE is a tool that is use for measuring the different levels of expression $[14,15]$.

ClustalW method is a method that is used to determine the sequences of amino acids of EIN3/EIL1 genes by aligning by using the sequences [14]. Then next hylogenetic relationships carefully taken by analysis with using the MEGA 6 software and neighbor joining method is used with poisson correction as well as replicate of 1000 bootstraps [16].

\section{Bioinformatics Analysis of EIN3/EIL1 genes}

Gene Ontology is the bioinformatics tool that is use to access the functional information about EIN3/EIL1 genes[17].KEGG(Kyoto Encyclopedia of Genes and Genomes) pathways of the EIN3/EIL1 genes is also bioinformatics tools that is functionally use for classification and characterization of genes at functional level with using the principles of functional enrichment analysis. Functional enrichment software that is used for classification and characterization of genes at functional level [18].

The interactions among proteins using bioinformatics software helpful for better understanding the functions of specific genes found in plant. Biological database such as STRING is designed for to construct the specific PPI networks of EIN3/EIL1 due to known PPIs and analysis to check interactions among proteins [19].

\section{Identification of Genes}

MapChart software is also used to find out the location of EIN3/EIL1 genes on chromosomes [20-22]. ExPASy software is use for examine the molecular mass, size and biochemical parameter such as isoelectric point of EIN3/EIL1 genes [22, 23]. WoLF PSORT software is also sue to predict the subcellular positions of EIN3/EIL1 genes. SMART software use to check the main domain of EIN3/EIL1 genes [24]. Biological database such as Phytozome database use for biochemical identification of specific sequences of EIN3/EIL1genes. Bioinformatics tool such as BLASTP use for identification of transcription factors of EIN3/EIL1genes [25, 26].

\section{Gene Expression Analysis}

Biological database such as Plant CARE is especially used to analyze the cis-elements in specific sequences of promoters of EIN3/EIL1 that are especially involved in transcription of genes [26-28]. psRNA Target is use to analyze the gene expression of miRNA target of the G.hirsutum EIN3/EIL1 [30].

As a highly important transcription factor, one crucial function of EIN3 is to recognize and bind to specific target genes. The Ecker group first showed that EIN3/EILs family proteins regulate gene expression by binding directly to a primary ethylene response element recently, they further identified the repertoire of EIN3 target genes, using ChIP-Seq and mRNA-Seq during a time course of ethylene treatment $[25,26]$.

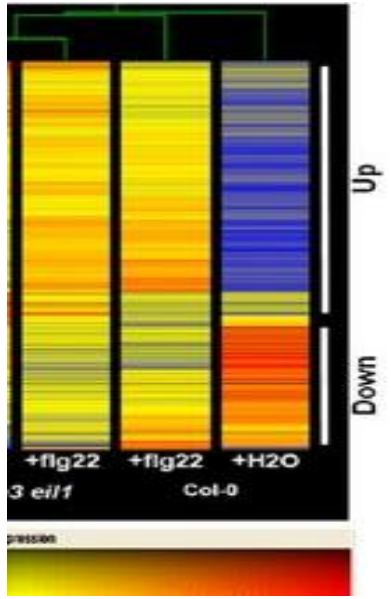

Fig-2: Gene expression analysis of EIN3-1 EIL1 genes upregulation
However, a central, yet unanswered question is how EIN3 recognizes and binds to a wide variety of target DNA. Previous studies have demonstrated that the N-terminal region of EIN3 (for instance, residues

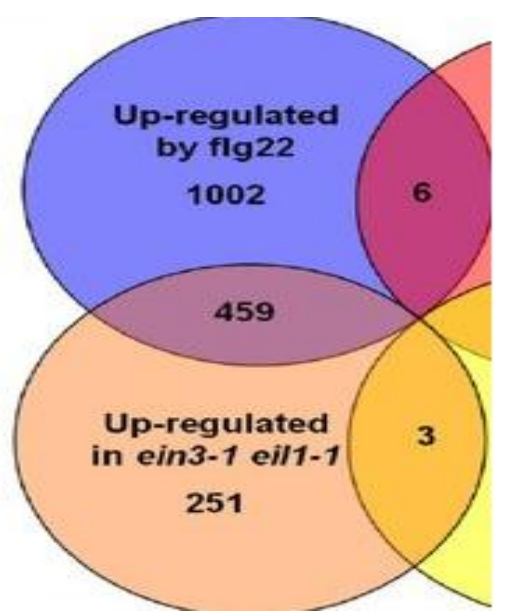

used by different studies, and also its homolog in tobacco TEIL is responsible for DNA binding [24, 30], while the optimal DNA-binding domain (DBD) and the core DBD of EIN3 are still unknown. In addition, EIN3 
was shown to bind specifically to the fragment of ERF1 promoter $(-1213$ to -1178$)$ which has two palindromic repeats flanking one central EIN3-binding site (EBS) [24]; yet except for ERF1, no other EIN3 target bearing palindromic repeat sequences has been reported. Thus, a crystal structure of EIN3 would still be desired for the inspection-at atomic level-of residues essential to the function of EIN3 [30]. Consequently, such limited knowledge regarding to the molecular mechanism of the interaction between EIN3 and DNA targets still significantly impede an in-depth view of transcriptional regulation initiated by EIN3.

\section{CONCLUSION}

Ethylene response is a target for a complex regulation, in which EIN3/EIL1 TFs play a crucial role. Recent studies shed light on multiple layers of complexity in tuning EIN3/EIL1 function (including epigenetic gene silencing and modulation of EIN3/EIL1 stability and activity via PPIs) that facilitate the "shaping" of ethylene response according to spatiotemporal and environmental conditions. It helpful in deigned in elucidating the role of EIN3 and EIL1genes for better understanding the molecular mechanism for the development of cotton fibre in future studies. Also in explaining the interactions of EIN3 and EIL1genes with different molecular pathways that are involved for better development of cotton fibre

\section{REFRENCES}

1. Cao, Y., Han, Y., Meng, D., Li, D., Jin, Q., Lin, Y., \& Cai, Y. (2017). Genome-wide analysis suggests high level of microsynteny and purifying selection affect the evolution of EIN3/EIL family in Rosaceae. PeerJ, 5, e3400.

2. Dai, X., Zhuang, Z., \& Zhao, P. X. (2018). psRNATarget: a plant small RNA target analysis server (2017 release). Nucleic acids research, 46(W1), W49-W54.

3. Dolgikh, V. A., Pukhovaya, E. M., \& Zemlyanskaya, E. V. (2019). shaping ethylene response: the role of EIN3/EIL1 transcription factors. Frontiers in Plant Science, 10, 1030.

4. Harkey, A. F., Watkins, J. M., Olex, A. L., DiNapoli, K. T., Lewis, D. R., Fetrow, J. S., ... \& Muday, G. K. (2018). Identification of transcriptional and receptor networks that control root responses to ethylene. Plant physiology, 176(3), 2095-2118.

5. Hu, Y., Vandenbussche, F., \& Van Der Straeten, D. (2017). Regulation of seedling growth by ethylene and the ethylene-auxin crosstalk. Planta, 245(3), 467-489.

6. Li, M., Wang, R., Liang, Z., Wu, X., \& Wang, J. (2019). Genome-wide identification and analysis of the EIN3/EIL gene family in allotetraploid Brassica napus reveal its potential advantages during polyploidization. BMC plant biology, 19(1), 110.

7. Maruyama-Nakashita, A., Nakamura, Y., Tohge,
T., Saito, K., \& Takahashi, H. (2006). Arabidopsis SLIM1 is a central transcriptional regulator of plant sulfur response and metabolism. The Plant Cell, 18(11), 3235-3251.

8. O’Malley, R. C., Huang, S. S. C., Song, L., Lewsey, M. G., Bartlett, A., Nery, J. R., ... \& Ecker, J. R. (2016). Cistrome and epicistrome features shape the regulatory DNA landscape. Cell, 165(5), 1280-1292.

9. Pathan, M., Keerthikumar, S., Chisanga, D., Alessandro, R., Ang, C. S., Askenase, P., ... \& Collino, F. (2017). A novel community driven software for functional enrichment analysis of extracellular vesicles data. Journal of extracellular vesicles, 6(1), 1321455.

10. Pawełkowicz, M. E., Skarzyńska, A., Cebula, J., Hincha, D., Ziąbska, K., Pląder, W., \& Przybecki, Z. (2017, August). Bioinformatics and expressional analysis of cDNA clones from floral buds. In Photonics Applications in Astronomy, Communications, Industry, and High Energy Physics Experiments 2017 (Vol. 10445, p. 1044538). International Society for Optics and Photonics.

11. Rieu, I., Mariani, C., \& Weterings, K. (2003). Expression analysis of five tobacco EIN3 family members in relation to tissue- specific ethylene responses. Journal of Experimental Botany, 54(391), 2239-2244.

12. Shi, H., Lyu, M., Luo, Y., Liu, S., Li, Y., He, H., ... \& Zhong, S. (2018). Genome-wide regulation of light-controlled seedling morphogenesis by three families of transcription factors. Proceedings of the National Academy of Sciences, 115(25), 64826487.

13. Song, J., Zhu, C., Zhang, X., Wen, X., Liu, L., Peng, J., ... \& Yi, C. (2015). Biochemical and structural insights into the mechanism of DNA recognition by Arabidopsis ETHYLENE INSENSITIVE3. PLoS One, 10(9), e0137439.

14. Szklarczyk, D., Franceschini, A., Wyder, S., Forslund, K., Heller, D., Huerta-Cepas, J., ... \& Kuhn, M. (2015). STRING v10: protein-protein interaction networks, integrated over the tree of life. Nucleic acids research, 43(D1), D447-D452.

15. Thyssen, G. N., Fang, D. D., Turley, R. B., Florane, C., Li, P., \& Naoumkina, M. (2014). Next generation genetic mapping of the Ligon-lintless-2 (Li 2) locus in upland cotton (Gossypium hirsutum L.). Theoretical and applied genetics, 127(10), 2183-2192.

16. Naeem, M., Ali, J., Hassan, M. Z., Arshad, B., Rao, M. H. I., Sarmad, M. S. K., ... \& Hassan, M. U. (2019). Novel Approach Towards DNA Barcoding as a Tool in Molecular Biologyand Biological Activities of Cyclotides with Particular Emphasizes at Molecular Level. In Biological Forum-An InternationalJournal (Vol. 11, No. 2, pp. 83-96).

17. Naeem, A., Saddique, S., \& Chand, S. A. (2019). 
Advancement and Future Directions towards Herbal Treatment for Various Diseases.

18. Ahmad, I., Khan, S., Naeem, M., Hayat, M., Azmi, U. R., Ahmed, S., ... \& Irfan, M. (2019). Molecular Identification of Ten Palm Species using DNA Fingerprinting. Int. J. Pure App. Biosci, 7(1), 46-51.

19. Ahmad, I., Khan, S., Naeem, M., Hayat, M., Azmi, U. R., Ahmed, S., ... \& Irfan, M. (2019). Molecular Identification of Ten Palm Species using DNA Fingerprinting. Int. J. Pure App. Biosci, 7(1), 46-51.

20. Naeem, M., Hayat, M., Qamar, S. A., Mehmood, T., Munir, A., Ahmad, G., ... \& Hussain, A. (2019). Risk factors, genetic mutations and prevention of breast cancer. Int. J. Biosci, 14(4), 492-496.

21. Shafiq, S., Adeel, M., Raza, H., Iqbal, R., Ahmad, Z., Naeem, M., ... \& Azmaai, U. R. (2019). Effects of Foliar Application of Selenium in Maize (Zea Mays L.) under Cadmium Toxicity. In Biological Forum-An International Journal. 11(2): 27-37.

22. Buchanan, J. T., Colvin, K. M., Vicknair, M. R., Patel, S. K., Timmer, A. M., \& Nizet, V. (2008). Strain-associated virulence factors of Streptococcus iniae in hybrid-striped bass. Veterinary microbiology, 131(1-2), 145-153.

23. Chen, D. F., Wang, K. Y., Geng, Y., Wang, J., Huang, X. L., \& He, M. (2011). Pathological changes in cultured channel catfish Ictalurus punctatus spontaneously infected with Streptococcus iniae. Diseases of aquatic organisms, 95(3), 203-208.

24. Doublet, P., Van Heijenoort, J., Bohin, J. P., \& Mengin-Lecreulx, D. (1993). The murI gene of Escherichia coli is an essential gene that encodes a glutamate racemase activity. Journal of
Bacteriology, 175(10), 2970-2979.

25. Hashimoto, A., Nishikawa, T., Oka, T., Takahashi, K., \& Hayashi, T. (1992). Determination of free amino acid enantiomers in rat brain and serum by high-performance liquid chromatography after derivatization with $\mathrm{N}$-tert.-butyloxycarbonyl-Lcysteine and o-phthaldialdehyde. Journal of Chromatography B: Biomedical Sciences and Applications, 582(1-2), 41-48.

26. Kieber, J. J., \& Ecker, J. R. (1994). Molecular and genetic analysis of the constitutive ethylene response mutation ctr1. In Plant molecular biology (pp. 193-201). Springer, Berlin, Heidelberg.

27. Wesley, S. V., Helliwell, C. A., Smith, N. A., Wang, M., Rouse, D. T., Liu, Q., ... \& Robinson, S. P. (2001). Construct design for efficient, effective and high- throughput gene silencing in plants. The Plant Journal, 27(6), 581-590.

28. Carbonell, A. U., Cho, C. H., Tindi, J. O., Counts, P. A., Bates, J. C., Erdjument-Bromage, H., ... \& Banne, E. (2019). Haploinsufficiency in the ANKS1B gene encoding AIDA-1 leads to a neurodevelopmental syndrome. Nature communications, 10(1), 1-15.

29. Zhang, T., Hu, Y., Jiang, W., Fang, L., Guan, X., Chen, J., ... \& Hulse-Kemp, A. M. (2015). Sequencing of allotetraploid cotton (Gossypium hirsutum L. acc. TM-1) provides a resource for fiber improvement. Nature biotechnology, 33(5), 531-537.

30. Zhang, X., Ji, Y., Xue, C., Ma, H., Xi, Y., Huang, P., ... \& Guo, H. (2018). Integrated regulation of apical hook development by transcriptional coupling of EIN3/EIL1 and PIFs in Arabidopsis. The Plant Cell, 30(9), 1971-1988. 